\title{
Nonlinear Transmission Lines for Pulse Shaping in Silicon
}

\author{
Ehsan Afshari, Student Member, IEEE, and Ali Hajimiri, Member, IEEE
}

\begin{abstract}
Nonlinear transmission lines (NLTL) are used for pulse shaping. We developed the theory of pulse propagation through the NLTL. The problem of a wide pulse degenerating into multiple pulses rather than a single pulse is solved by using a gradually scaled NLTL. We exploit certain favorable properties of accumulation-mode MOS varactors to design an NLTL that can simultaneously sharpen both rising and falling edges. There is a good agreement among the theory, simulations, and measurements.
\end{abstract}

Index Terms-Accumulation-mode varactors, edge sharpening, nonlinear effects, nonlinear transmission lines, pulse shaping, solitons, transmission lines.

\section{INTRODUCTION}

$\mathbf{T}$ HE concept of a solitary wave was introduced to science by John Scott Russell 170 years ago [1]. In 1834 he observed a wave which was formed when a rapidly drawn boat came to a sudden stop in narrow channel. According to his diary, this wave continued "at great velocity, assuming the form of a large solitary elevation, a well-defined heap of water that continued its course along the channel apparently without change of form or diminution of speed." These solitary waves, now called solitons, have become important subjects of research in diverse fields of physics and engineering. There is a considerable body of work on solitons in applied mathematics (e.g., [2], [3]), applied physics—especially in optics (e.g., [4]-[7])—and a few works in electronics [8]-[10]. The ability of solitons to propagate with small dispersion can be used as an effective means to transmit data, modulated as short pulses over long distances; one example of this is the ultra wideband impulse radio that has recently gained popularity [16].

An important related application is pulse sharpening for the more traditional nonreturn-to-zero (NRZ) data transmission in digital circuits by improving the edges of the pulses. Improving the transitions by shrinking the rise and fall times of pulses can be useful in other applications, such as high-speed sampling and timing systems. Nonlinear transmission lines (NLTLs) sharpening of either the rising or falling edge of a pulse has been demonstrated on a GaAs technology [9], [10]. However, to the best of our knowledge, to this date there has been no demonstration of simultaneous reduction of both rise and fall times in an NLTL. Neither are we aware of any demonstration of such NLTLs in silicon-based CMOS process technologies.

Manuscript received May 20, 2004; revised September 10, 2004. This work was supported by the Lee Center and the National Science Foundation.

The authors are with the California Institute of Technology, Pasadena, CA 91125 USA (e-mail: ehsan@caltech.edu).

Digital Object Identifier 10.1109/JSSC.2005.843639
In this work, we first show a soliton line on a conventional silicon technology, which can achieve very narrow pulses with a bandwidth in excess of the cut-off frequency $f_{T}$ of the fastest transistor in this process. Next, we demonstrate that using a favorable characteristic of MOS varactors, which leads to a different kind of nonlinearity, we can improve both the rise and fall times, simultaneously. This is not possible with the nonlinear elements commonly used in the NLTLs (e.g., reverse bias PN junctions). Neither can it be done using transistors, as they are limited by their unity gain frequency, $f_{T}$.

In this paper, the propagation of soliton waves in a nonlinear transmission line will be studied. We introduce two different types of nonlinear transmission lines to generate narrow pulses and to sharpen pulse transitions, respectively. Finally, we show the experimental results verifying the agreement between the theory and the measurement.

\section{THEORY OF NONLINEAR TRANSMISSION LINE}

In this section we review the basic theory behind nonlinear transmission lines and their use for pulse narrowing and edge sharpening in Sections II-A and II-B, respectively. Fig. 1 shows an example of a nonlinear transmission line using inductors, $l$, and voltage-dependent (hence nonlinear) capacitors, $c(V)$.

By applying $K C L$ at node $n$, whose voltage with respect to ground is $V_{n}$, and applying $K V L$ across the two inductors connected to this node, as shown in [15], one can easily show that voltages of the adjacent nodes on this NLTL are related via

$$
l \frac{d}{d t}\left[c\left(V_{n}\right) \frac{d V_{n}}{d t}\right]=V_{n+1}+V_{n-1}-2 V_{n} .
$$

The right-hand side of (1) can be approximated with partial derivatives with respect to distance, $x$, from the beginning of the line, assuming that the spacing between two adjacent sections is $\delta$ (i.e., $x_{n}=n \delta$.) An approximate continuous partial differential equation can be obtained by using the Taylor expansions of $V(x-\delta), V(x)$, and $V(x+\delta)$, as shown in the Appendix, to evaluate the right-hand side of (1), i.e.,

$$
\ell \frac{\partial}{\partial t}\left[c(V) \frac{\partial V}{\partial t}\right]=\delta^{2} \frac{\partial^{2} V}{\partial x^{2}}+\frac{1}{12} \delta^{4} \frac{\partial^{4} V}{\partial x^{4}} .
$$

Defining $L=\ell / \delta$ and $C(V)=c(V) / \delta$ as the inductance and capacitance per unit length, respectively, we write (2) as

$$
L \frac{\partial}{\partial t}\left[C(V) \frac{\partial V}{\partial t}\right]=\frac{\partial^{2} V}{\partial x^{2}}+\frac{\delta^{2}}{12} \frac{\partial^{4} V}{\partial x^{4}} .
$$




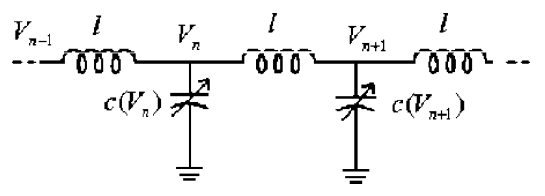

Fig. 1. Nonlinear transmission line.

It is noteworthy that for a continuous transmission line $(\delta \rightarrow 0)$, (3) reduces to

$$
L \frac{\partial}{\partial t}\left[C(V) \frac{\partial V}{\partial t}\right]=\frac{\partial^{2} V}{\partial x^{2}}
$$

In a linear transmission line when $C(V)=C=$ const., (4) can be written as

$$
\frac{\partial^{2} V}{\partial t^{2}}-\frac{1}{L C} \frac{\partial^{2} V}{\partial x^{2}}=0
$$

\section{A. Pulse Narrowing Nonlinear Transmission Lines}

In this section, we approximate the capacitor's voltage dependence using the following first-order linear approximation:

$$
C(V)=C_{o}(1-b V)
$$

where $C_{0}$ and $b$ are constants. In this case, (3) reduces to

$$
\frac{\partial^{2} V}{\partial t^{2}}-\frac{1}{L C_{o}} \frac{\partial^{2} V}{\partial x^{2}}=\frac{\delta^{2}}{12} \frac{1}{L C_{o}} \frac{\partial^{4} V}{\partial x^{4}}+\frac{b}{2} \frac{\partial^{2}\left(V^{2}\right)}{\partial t^{2}}
$$

where the left-hand side is the classic wave equation, and the first and second terms on the right-hand side represent dispersion and nonlinearity, respectively.

If the effect of the dispersive and nonlinear terms in (7) are on the same order of magnitude, it is possible to have a single pulse solution for (7) with a profile that does not change as it propagates with velocity, $v$. A propagating mode solution can be obtained by converting the partial differential equation (PDE) of (7) to an ordinary differential equation (ODE) by a simple change of variable, $u=x-v t$. The complete derivation can be found in [15]. This solution is

$$
V(x, t)=\frac{3\left(v^{2}-v_{0}^{2}\right)}{b v^{2}} \operatorname{sech}^{2}\left[\frac{\sqrt{3\left(v^{2}-v_{0}^{2}\right)}}{v_{0}} \frac{(x-v t)}{\delta}\right]
$$

where $v$ is the propagation velocity of the pulse and $v_{0}=1 / \sqrt{L C_{0}}$. It can be proven mathematically that (8) is the only physically meaningful traveling wave solution to (7) that maintains its shape while propagating through NLTL. This solution is shown in Fig. 2 for three different values of $L$ and $C$, and hence different $\delta$. Note that this solution is not a function of the input waveform, and thus any arbitrary input will eventually turns into (8), if it goes through a line which is long enough.

As can be seen from (8), the peak amplitude is a function of the velocity. Defining an effective capacitance, $C_{\text {eff }}$, so that $v=1 / \sqrt{L C_{\text {eff }}}$, the pulse height is given by

$$
V_{\max }=\frac{3}{b} \cdot \frac{v^{2}-v_{0}^{2}}{v^{2}}=\frac{3}{b}\left(1-\frac{C_{\mathrm{eff}}}{C_{o}}\right) .
$$

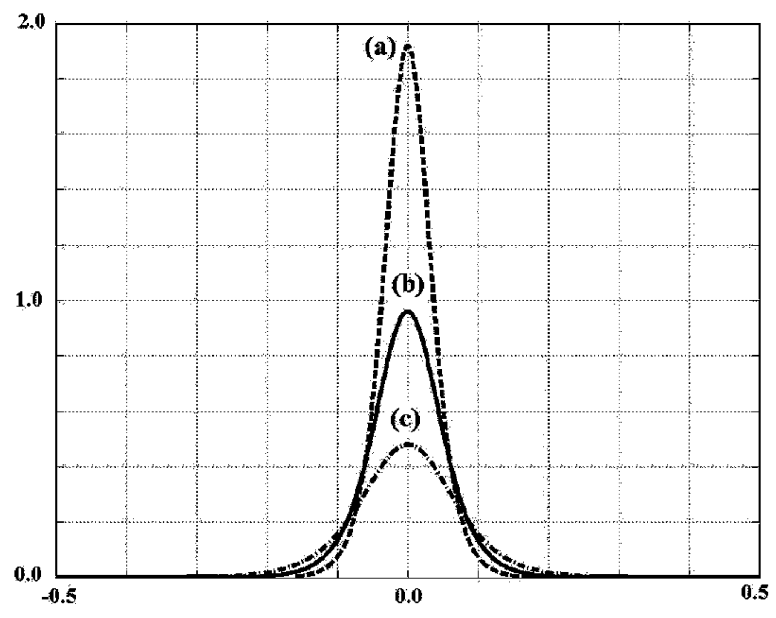

Fig. 2. Three normalized soliton shapes for different values of $L$ and $C$ (a) $L=1 \mathrm{nH}$ and $C=1 \mathrm{nF}$. (b) $L=2 \mathrm{nH}$ and $C=2 \mathrm{nF}$. (c) $L=4 \mathrm{nH}$ and $C=4 \mathrm{nF}$.

Using (9), we can relate $C_{\text {eff }}$ to an effective voltage $V_{\text {eff }}$. It is straightforward to show that

$$
V_{\mathrm{eff}}=\frac{V_{\mathrm{max}}}{3} .
$$

So it is the capacitance at one-third the peak amplitude that determines the effective propagation velocity. Using (8)-(10) we can easily calculate the half-height width of the pulse to be

$$
W \approx \frac{\delta}{v} \frac{v_{0}}{\sqrt{\left(v^{2}-v_{0}^{2}\right)}} .
$$

As can be seen, in a weakly dispersive and nonlinear transmission line, the nonlinearity can counteract the normally present dispersive properties of the line maintaining solitary waves that propagate without dispersion. This behavior can be explained using the following intuitive argument. The instantaneous propagation velocity at any given point in time and space is given by $1 / \sqrt{L C}$. In the presence of a nonlinear capacitor with a characteristic given by (6), the instantaneous capacitance is smaller for higher voltages. Therefore, the points closer to the crest of the voltage waveform experience a faster propagation velocity and produce a shock-wave front, due to the nonlinearity, as shown symbolically in the upper part of Fig. 3. Note that this is not a real waveform and more a fictitious representation of how each point on the curve tends to evolve. On the other hand, dispersion of the line causes the waveform to spread out, as shown in the lower half of Fig. 3. For a proper nonlinearity determined by (7), these two effects can cancel each other out.

A few important observations are: 1) the velocity of the solitary wave increases with its amplitude; 2) pulse width decreases with increasing pulse velocity; 3) the width shrinks for higher amplitudes; and 4) the sign of solution depends on the sign of nonlinearity factor, $b$, i.e. for a capacitor with a positive voltage dependence (e.g., an nMOS varactor in accumulation mode) we have

$$
C(V)=C_{o}(1+b V)
$$

resulting in upside-down pulses. 


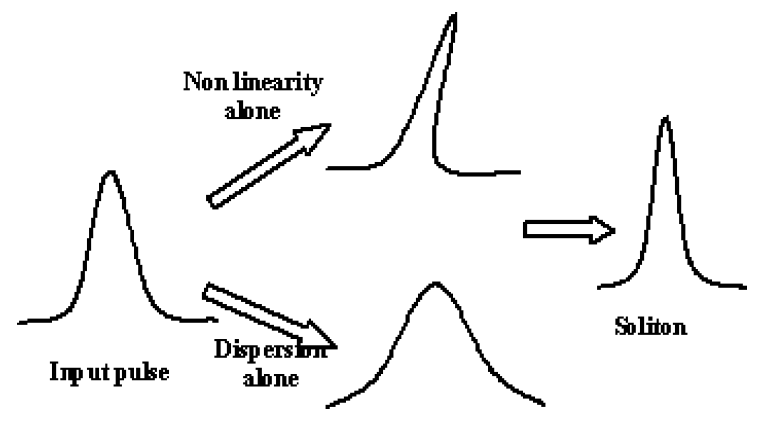

Fig. 3. Dispersion and nonlinear effects in the NLTL.

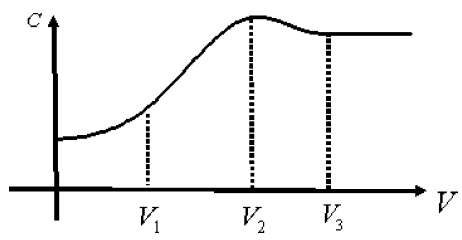

Fig. 4. Capacitance versus voltage for a MOSVAR.

Based on these results, to achieve large-amplitude narrow pulses, inductance and capacitance of the NLTL must be as small as possible, and nonlinearity factor, $b$, should be large enough to compensate the dispersion of the line.

It is also important to know the characteristic impedance of these lines (for impedance matching, etc.). As in a NLTL the capacitance is a function of voltage, we can only define an effective semi-empirical value for the characteristic impedance. Simulation results indicate that one can approximate $Z_{\text {eff }}$ using the capacitance at $V_{\text {eff }}$ defined in (13), i.e.,

$$
Z_{\text {eff }}=\sqrt{\frac{L}{C\left(V_{\max } / 3\right)}} .
$$

\section{B. Edge Sharpening Lines}

It is possible to design NLTLs to sharpen the pulse transitions. This is particularly useful for digital transmission such as NRZ data. Unfortunately, all the efforts in the past [6] have resulted in sharpening of only one of the rising and falling edges. This, however, has very little practical value, as both transitions are equally important in common NRZ digital systems. This problem can be traced back to the monotonic dependence of the nonlinear capacitive elements used in NLTL on the voltage [e.g., reverse biased PN junction, or the ideal behavior of (6) and (12)].

Fortunately, CMOS processes offer different characteristics for nonlinear capacitors that can be exploited to achieve simultaneous edge sharpening for both rising and falling edges. More specifically, accumulation-mode MOS varactors [11] (an nMOS capacitor in an n-well) offer nonmonotonic voltage dependence. Particularly, the secondary reduction of capacitance shown in Fig. 4 due to poly-silicon depletion [12], [13] and short-channel charge quantization [13] effects can be used for edge sharpening.

Fig. 5 shows symbolically how one can use the behavior of Fig. 4 to sharpen both edges. First, let us focus on the rise-

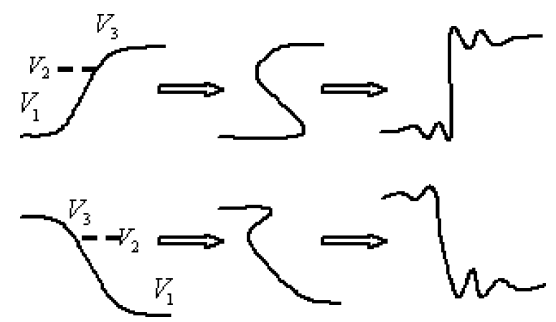

Fig. 5. How rise and fall time vary within the NLTL.

time reduction. Consider the rising edge shown in the upper part of Fig. 5. Initially the voltage is low, which corresponds to a smaller capacitance per Fig. 4, and hence a faster instantaneous propagation velocity for the lower end of the pulse. As the voltage goes up, the capacitance increases, resulting in a decrease in the instantaneous propagation velocity. This pushes the lower end of the transition forward in time and results in sharpening of the rising edge. This effect is symbolically shown in the fictitious middle waveform of Fig. 5. The fall time reduction can be explained using the lower part of Fig. 5. This is where the nonmonotonic behavior of Fig. 4 plays its role. The upper part of the transition (voltages above $V_{2}$ ) will be accelerated due to the reduction of the capacitance and will create an advancing front, as symbolically shown in the middle waveform of Fig. 5. The lower capacitance at the very low voltages can generate a leading tail, which will be partially dissipated by the line. The weak reduction in capacitance from $V_{2}$ to $V_{3}$ versus reduction from $V_{1}$ to $V_{2}$ results in mismatched rise/fall time as can be seen in Figs. 5 and 11.

While the above explanation based on a simplified memoryless description of the line provides a basic intuition for its operation, a complete description can only be obtained by solving the differential equation in (3) to account for the memory of the system. ${ }^{1}$ Our numerical solution of (6) also confirms that as long as the input voltage range exceeds voltages, $V_{1}$ and $V_{3}$, for a range of $L$ 's and $C$ 's, the line sharpens both rising and falling edges, simultaneously.

It may also be possible to achieve a symmetrical wave form in the following ways.

1) Using an n-type and a p-type MOSVAR in parallel to create a symmetrical $C(V)$ curve. The problem with this method is that a p-type MOSVAR is not as fast as n-type MOSVAR, therefore, the frequency response of the line would be limited to the frequency response of the p-type MOSVARs.

2) Using two n-type MOSVAR at each node, as shown in Fig. 6. This way, we can have a symmetrical $C(V)$ curve, however the capacitance of each node would be twice as large which limits the cut-off frequency of the line by a factor of 1.4. Another limitation of this method is the additional parasitic capacitance to the substrate that may lower the effective nonlinearity factor, $b$, of the capacitors.

${ }^{1}$ We hypothesize that other dynamic effects in the MOS varactor may also help edge sharpening, e.g., the processes of charge being attracted from the n+ diffusions to the channel and repelling them are not exact inverses of each other over short time intervals. Some of the repelled accumulation charges will be absorbed inside the well. This changes the response time of the capacitor and keeps it higher for a longer period of time for the falling edge. 


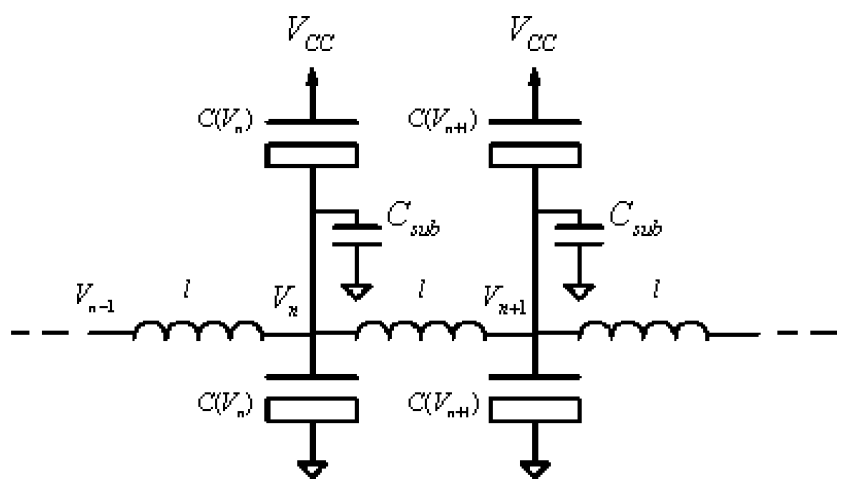

Fig. 6. Proposed NLTL for symmetrical edge sharpening.

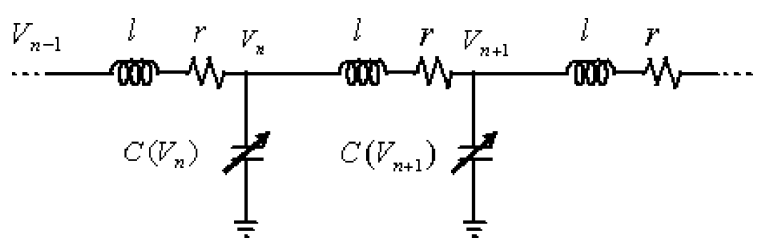

Fig. 7. Simple model of a lossy nonlinear transmission line.

In this work, the goal was to achieve the minimum rise time while decreasing the fall time at the same time, so we used a single capacitor at each node. For other applications with different objectives one of these alternative methods may be preferred.

\section{EFFECT OF LOSS}

Fig. 7 shows a simple model of a lossy nonlinear transmission line. By applying $K C L$ at node $n$, whose voltage with respect to ground is $V_{n}$, and applying $K V L$ across the two branches connected to this node, as shown in [15], one can easily show that voltages of adjacent nodes on this NLTL are related via

$$
V_{n-1}-2 V_{n}+V_{n+1}=l \frac{d}{d t}\left(I_{n-1}-I_{n}\right)+r\left(I_{n-1}-I_{n}\right)
$$

where $r$ is the resistance of each section.

An approximate continuous partial differential equation can be obtained similar to (2) as

$$
\frac{\partial^{2} V}{\partial x^{2}}+\frac{\delta^{2}}{12} \frac{\partial^{4} V}{\partial x^{4}}=L \frac{\partial}{\partial t}\left[\left(C(V) \frac{\partial V}{\partial t}\right)\right]+R C(V) \frac{\partial V}{\partial t} .
$$

Unfortunately, we could not find an analytical solution for (15) and had to use numerical methods to solve it.

Another model for the loss of the transmission line is shown in Fig. 8. In this case one can show that the governing equation of the line is

$$
\frac{\partial^{2} V}{\partial t^{2}}-b \frac{\partial^{2} V^{2}}{\partial t^{2}}=v_{0}^{2}\left[\frac{\partial^{2} V}{\partial x^{2}}+\frac{1}{12} \frac{\partial^{4} V}{\partial x^{4}}\right]+\frac{R}{L}\left[\frac{\partial^{2} V}{\partial x^{2}}-b \frac{\partial^{2} V^{2}}{\partial x^{2}}\right]
$$

which can be reduced to Burgers equation [14], [15] as shown in [15].

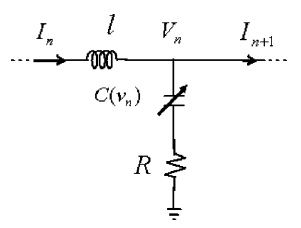

Fig. 8. Simple model of a lossy nonlinear transmission line.
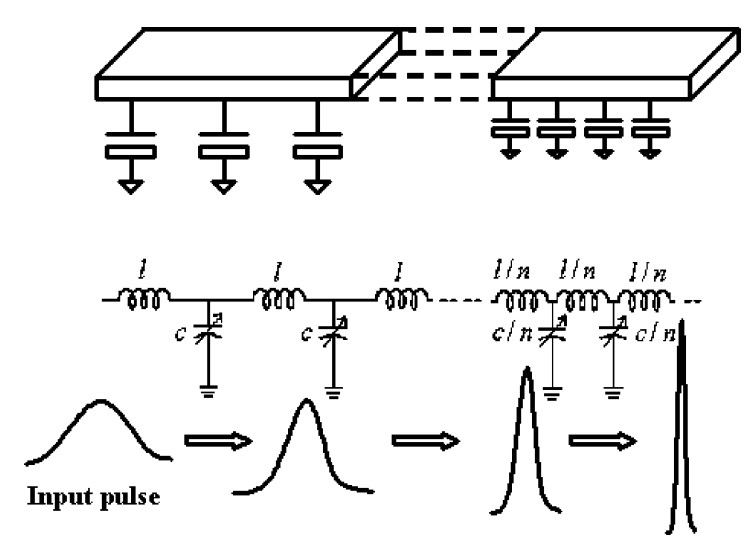

Fig. 9. Schematic of the gradually scaled nonlinear transmission line.

In both models, the numerical solution of the governing equations shows that loss has an effect similar to the dispersion, meaning that loss causes the waveform to spread out, so in order to have a soliton pulse in a lossy nonlinear transmission line, nonlinearity should be strong enough to cancel out both dispersion and loss.

\section{GRADUALLY SCALED NLTL}

One problem in pulse narrowing NLTLs is that if the input pulse is wider than a certain minimum related to the natural pulse width of the line in (11), the line is incapable of concentrating all that energy into one pulse and instead the input pulse degenerates into multiple soliton pulses, as shown in the simulated upper waveforms of Fig. 10. This is an undesirable effect that cannot be avoided in a standard line.

We can solve this problem by using gradually scaled nonlinear transmission lines [9]. We notice that the characteristic pulse width of the line is controlled by the node spacing, $\delta$, and the propagation velocity, $v$, which is in turn controlled by $L$ and $C$. Thus, we use a gradual line consisting of several segments that are gradually scaled to have smaller characteristic pulse width, as shown in Fig. 9.

The first few segments have the widest characteristic pulse, meaning that their output is wider and has smaller amplitude. As a result, the input pulse will cause just one pulse at the output of these segments. The following segments have a narrower response and the last segment has the narrowest one. This will guarantee the gradual narrowing of the pulses and avoids degeneration. Each segment has to be long enough so that the pulse can reach the segment's steady-state response before entering the next segment.

One design consideration is that the characteristic impedance of each segment matches those of the adjacent segments to avoid reflections. This requires the same scaling factor for both $L$ and 


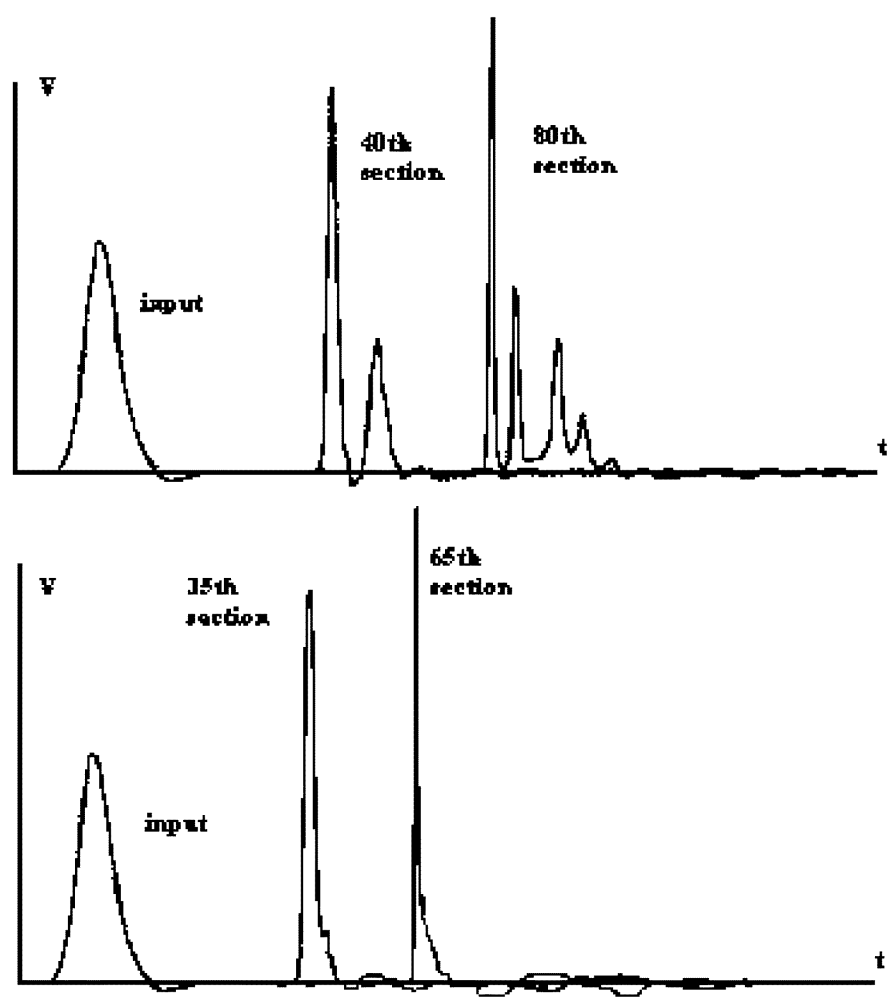

Fig. 10. Output waveforms of the normal and gradual soliton line.

$C$, so that their ratio remains constant. If we assume a linear approximation for $C-V$ curve of the voltage variable capacitors, we could mathematically model the scaled inductors and variable capacitors as

$$
C\left(x_{n}, V_{n}\right)=C_{0}\left(x_{n}\right)\left(1-b V_{n}\right)
$$

where $C_{0}\left(x_{n}\right)=C_{0}\left(1-a_{1} x_{n}\right)$ and

$$
L\left(x_{n}\right)=L_{0}\left(1-a_{2} x_{n}\right)
$$

where $L_{0}$ and $C_{0}$ represent the inductance and $0 \mathrm{~V}$ bias capacitance of the input stage respectively, $x_{n}$ is the distance from the input node, and $a_{1}$ and $a_{2}$ are the tapering factor of the capacitors and inductors, respectively. Here the assumption is that each section is scaled compared to its previous one and $a_{1}$ and $a_{2}$ are rate of the scaling of capacitors and inductors, respectively. That is, we have a NLTL with no two adjacent sections at the same scale. Now we can write a wave equation for a gradually scaled NLTL by plugging (17) and (18) into (3):

$$
\begin{gathered}
L\left(x_{n}\right) \frac{\partial}{\partial t}\left[C\left(x_{n}, V_{n}\right) \frac{\partial V_{n}}{\partial t}\right] \\
=\frac{\partial^{2} V_{n}}{\partial x^{2}}+\frac{1}{12} \delta^{2} \frac{\partial^{4} V_{n}}{\partial x^{4}} \\
L_{0}\left(1-a_{2} x_{n}\right) \frac{\partial}{\partial t}\left[C_{0}\left(1-a_{1} x_{n}\right)\left(1-b V_{n}\right) \frac{\partial V_{n}}{\partial t}\right] \\
=\frac{\partial^{2} V_{n}}{\partial x^{2}}+\frac{1}{12} \delta^{2} \frac{\partial^{4} V_{n}}{\partial x^{4}}
\end{gathered}
$$

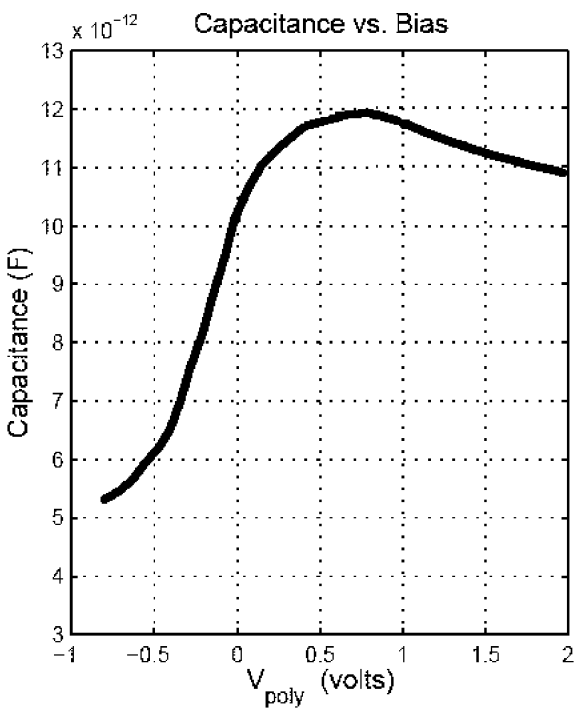

Fig. 11. Measured characteristic of MOSVAR used in the line.

assuming $a_{1} L \ll 1$ and $a_{2} L \ll 1$, where $L$ is the length of the line, we can simplify the above equation to

$$
\begin{aligned}
\frac{\partial^{2} V}{\partial t^{2}}- & \frac{1}{L_{0} C_{0}\left(1-\left(a_{1}+a_{2}\right) x\right)} \frac{\partial^{2} V}{\partial x^{2}} \\
& =\frac{\delta^{2}}{12} \frac{1}{L_{0} C_{0}\left(1-\left(a_{1}+a_{2}\right) x\right)} \frac{\partial^{4} V}{\partial x^{4}}+\frac{b}{2} \frac{\partial^{2} V^{2}}{\partial t^{2}}
\end{aligned}
$$

we can use numerical methods to solve the PDE in (21). Under the assumption that $\left(a_{1}+a_{2}\right) L \ll 1$, one can approximate (21) and obtain the width of the pulse as

$$
W=\left(1-\frac{2 b V_{\max }}{3}\right) \sqrt{\frac{3 L_{0} C_{0}(1-k x)}{2 b V_{\max }}} .
$$

Based on (22), as a pulse travels along the line ( $x$ increases), its width will decrease. The waveforms of this gradually scaled NLTL are shown in the lower part of Fig. 10, demonstrating the effectiveness of this technique. It is noteworthy that this gradually scaling technique is also applicable to the edge sharpening lines and does improve their performance, as well.

\section{Simulation}

We have designed one edge sharpening and two pulse narrowing NLTLs with different tapering factors $\left(a_{1}\right.$ and $\left.a_{2}\right)$ using the accumulation-mode MOS varactors and metal micro-strip transmission lines in a $0.18-\mu \mathrm{m}$ BiCMOS process. Fig. 11 shows the measured characteristic of the accumulation-mode MOSVAR used in this design. All the capacitors have similar $C$ - $V$ characteristics; however, we used different capacitances along the line in order to build a gradually scaled NLTL.

To achieve the lowest pulse width in the pulse narrowing lines or the shortest rise and fall times in the edge sharpening line, it is necessary to carefully select the dc level and the voltage swing. In general, this may be an additional constraint in system design since it will require additional dc level shifting and amplification or attenuation to adjust the input levels. Nonetheless, this level of signal conditioning is easily achieved in today's integrated 


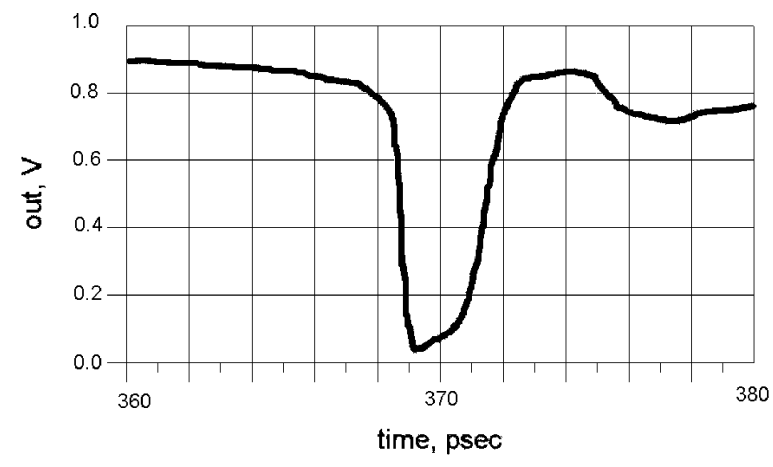

Fig. 12. Simulated output waveform of the pulse narrowing line.

circuits. The dc level and the voltage swing for each application is mentioned in the following sections.

All three lines consist of one hundred capacitors and one hundred inductors. We simulated the passive transmission lines in Sonnet [17] and the complete NLTL in ADS [18]. Next, we will discuss the details specific to each kind of lines separately.

\section{A. Pulse Narrowing Lines}

For pulse narrowing lines, we would like to have the maximum change in the capacitance with voltage. Thus, we chose the baseline dc bias point at $0.8 \mathrm{~V}$ which corresponds to the maximum capacitance point, and applied negative input pulses from this dc level. For a typical pulse amplitude of $1 \mathrm{~V}$, the effective nonlinearity factor $b$ in (12) is around $0.5 \mathrm{~V}^{-1}$. As we explained in Section IV, the lines are not continuously scaled, but consist of several segments with constant values of inductors and capacitors within a segment. (However it turns out that a continuous scaling of the line is preferable because of internal reflections between different segments of the line due to mismatch). The inductances and capacitances within each segment are lower than those of the previous segment. One of the lines consists of three different segments and the other of four. The results reported in this subsection and Section VI are those associated with the four-segment line which has a smaller pulse width. The lines are designed in such a way that the characteristic pulse width of each segment [given by (11)] is half that of the previous segment so the line can at least compress the input pulse by a factor of sixteen without degenerating into multiple pulses.

The simulated output waveform of the line to a $65 \mathrm{ps}$ wide input pulse is shown in Fig. 12. The simulation predicts that this silicon-based NLTL can produce negative pulses as narrow as $2.5 \mathrm{ps}$ (half amplitude width) with a $0.8 \mathrm{~V}$ amplitude at the output. It is noteworthy that transistors in this process are incapable of producing pulses nearly as narrow as those generated by the NLTL.

\section{B. Edge Sharpening Lines}

As we showed in the Section II-B, to build an edge sharpening line we need take advantage of the non monotonic $C-\mathrm{V}$ behavior exemplified by the secondary reduction in the capacitance, as shown in Fig. 11. Computer simulations show the best bias point and voltage swing are around -0.25 and $2 \mathrm{~V}$ at the input, respectively. Although these levels led to the best

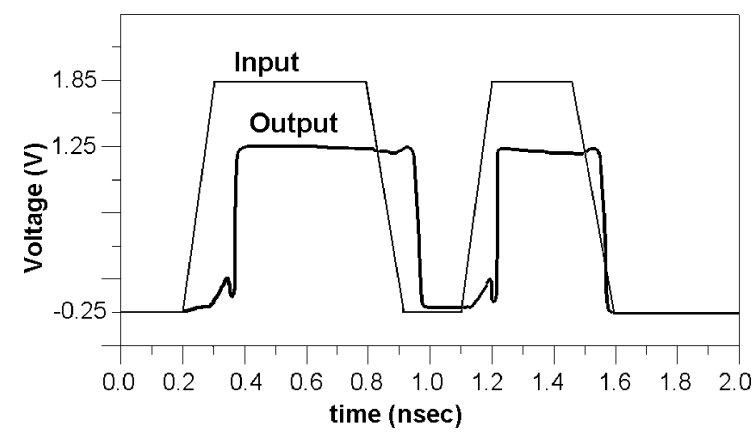

Fig. 13. Simulated input and output waveforms of the edge sharpening line.

achievable improvement in the rise and fall times, the line still enhances the rising and falling edges for input signal voltage swings between 1.5 and $2 \mathrm{~V}$. Fig. 13 shows the simulated input and output waveforms of this line.

The output pulses exhibit reduced rise and fall times of 1.5 and $20 \mathrm{ps}$, respectively. The rise and fall times of the output pulses are different because of the asymmetrical behavior of the nonlinear element for two different edges. We have also simulated this line with a pseudo-random data source and verified its edge sharpening functionality for any arbitrary data sequence. ${ }^{2}$

Unfortunately in this line, we cannot fully control the characteristic impedance of the lines because we have to pick the lowest capacitance and inductance-limited by the parasitic elements - to obtain that maximum improvement in the rise and fall times. This will allow us to maximize the cut-off frequency of the line. However, it is not possible to build very small nonlinear capacitors, because if we shrink the size of the accumulation-mode MOSVARs the effect of the parasitic capacitors becomes more important. These parasitic capacitors are voltage independent, hence linear, and will result in an effective reduction of the non linearity factor, $b$, in (12). In this design, the effective input impedance of the edge sharpening line is around $20 \Omega$ gradually scales to $50 \Omega$ at the output. So the input reflection coefficient of the line is roughly 0.4 . We must take this effect into account to be able to match the simulation and the measurement results.

\section{EXPERIMENTAL RESULTS}

All three lines were fabricated in a $0.18-\mu \mathrm{m}$ BiCMOS technology. Fig. 14 shows a chip microphotograph. We use RF probes to apply input to the line and to measure its output waveform. A $50 \mathrm{GHz}$ sampling oscilloscope is used to measure the input and output waveforms. A $k$-connector system of probes, connectors, and cables with a bandwidth of approximately $40 \mathrm{GHz}$ is used to bring the data to the oscilloscope. The main challenge in this measurement is the low bandwidth of the measurement system compared to the signal bandwidth, so it is essential to characterize the measurement setup carefully.

First, the oscilloscope was characterized using a signal source. We swept the source frequency and measured the amplitude of the signal on the oscilloscope, then using the same signal source, cables, and connectors, we measured the signal

${ }^{2}$ There seems to be some data dependant delay due to the nonlinear behavior of the lines in the simulations (see Fig. 13). This could have some implications for the data dependant jitter in the lines, which merits further studies. 


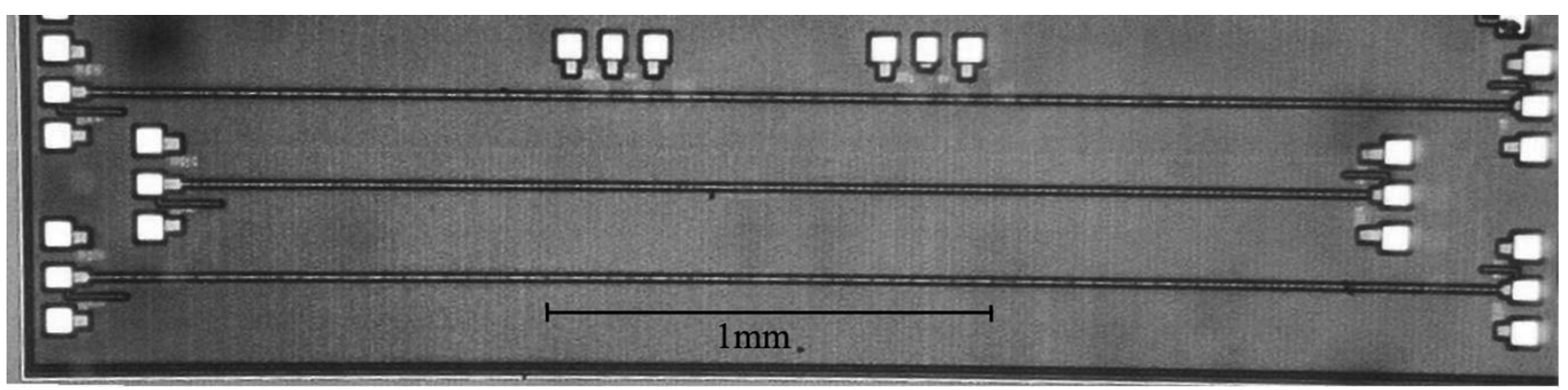

Fig. 14. Chip microphotograph: the middle line is an edge sharpening line and the other two are pulse narrowing lines.

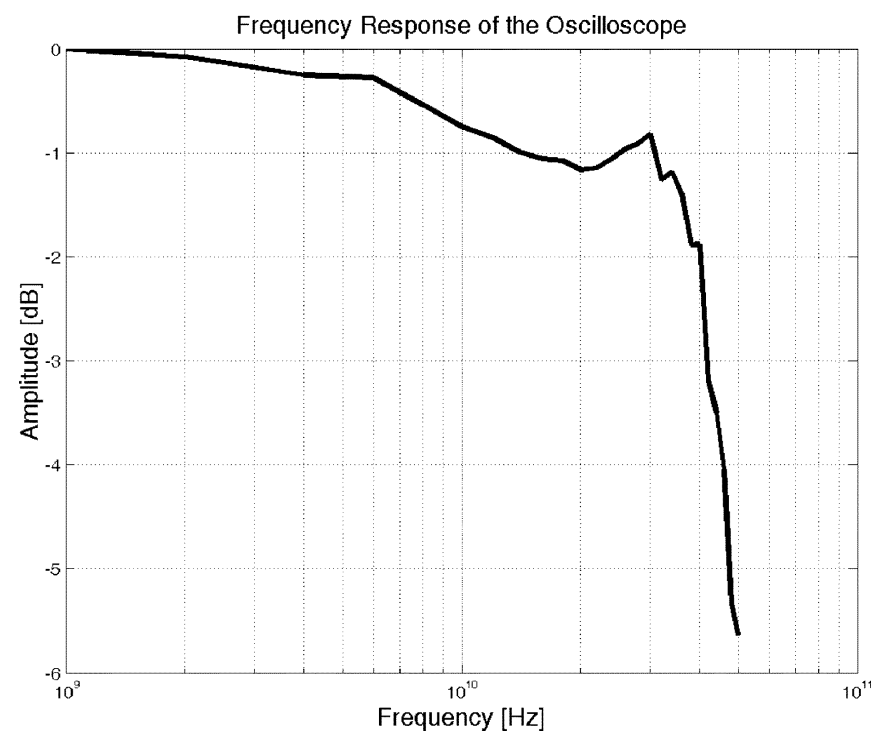

Fig. 15. Response of the oscilloscope.

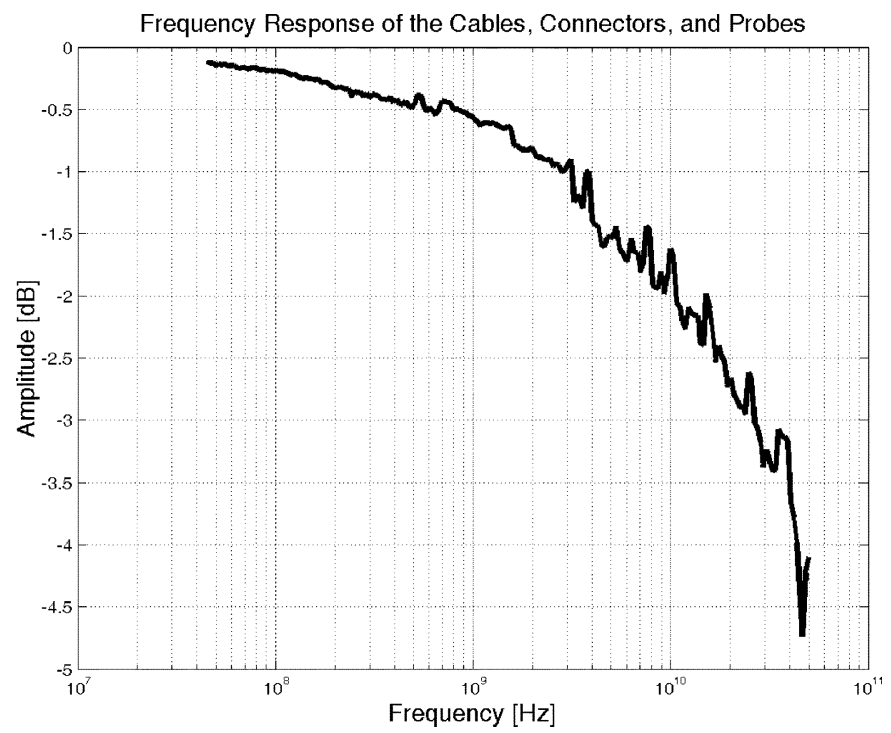

Fig. 16. Response of the cables, connectors, and probes.

amplitude using a wideband power meter. The ratio of these two values is the amplitude response of the oscilloscope. Fig. 15 shows this response. Then we characterize all other cables, connectors, probes, and bias tees using a $50 \mathrm{GHz}$ network analyzer. The response of these parts is shown in Fig. 16.

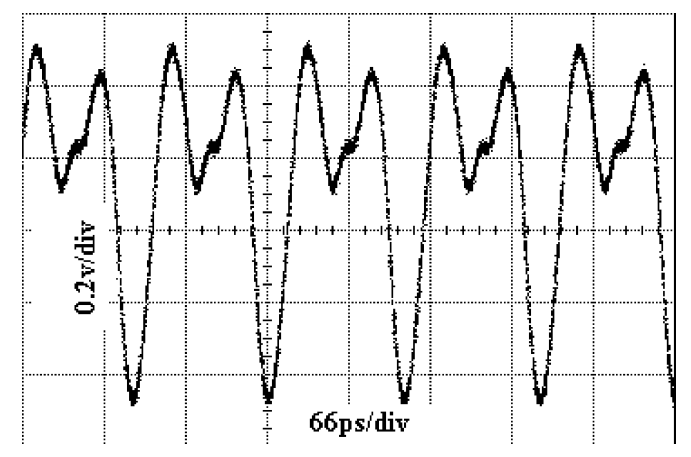

Fig. 17. Input and output of pulse narrowing line.

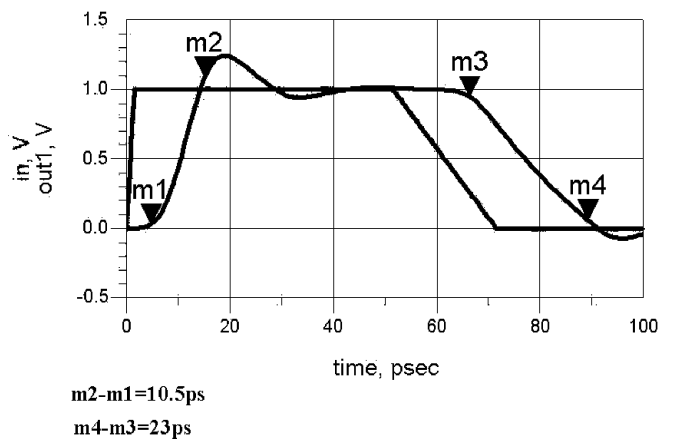

Fig. 18. Response of the measurement setup to an ideal input.

The amplitude response of the entire measurement setup is the product of Figs. 15 and 16. Using Matlab [19], one can show that the $10 \%-90 \%$ rise-time of such system is around $10.5 \mathrm{ps}$, which indicates that it is not possible to resolve rise times lower than $10.5 \mathrm{ps}$ and pulse widths lower than $21 \mathrm{ps}$.

Fig. 17 shows the measured response of the pulse narrowing line to a $50 \mathrm{ps}$ input pulse. Based on response of the measurement setup (Figs. 15 and 16), the response of the measurement setup to a $2.5 \mathrm{ps}$ pulse is $21.5 \mathrm{ps}$ wide. The measured pulse width is $22 \mathrm{ps}$, which is in good agreement with the simulation.

Matlab simulations show that if we have an ideal pulse with rise and fall times of $1.5 \mathrm{ps}$ and $20 \mathrm{ps}$, we should expect rise and fall times of $10.5 \mathrm{ps}$ and $23 \mathrm{ps}$, respectively, with this measurement setup, as it is shown in Fig. 18. The measured rise and fall times for this line are 11 ps and 25 ps, as shown in Fig. 19. Also it is important to note that the rise and fall times do not change with the input amplitude, as shown in Fig. 20, which verifies the nonlinear behavior of the line.

In the end, it is important to notice that we can set an upper bound for the pulse width of output pulses of our pulse nar- 


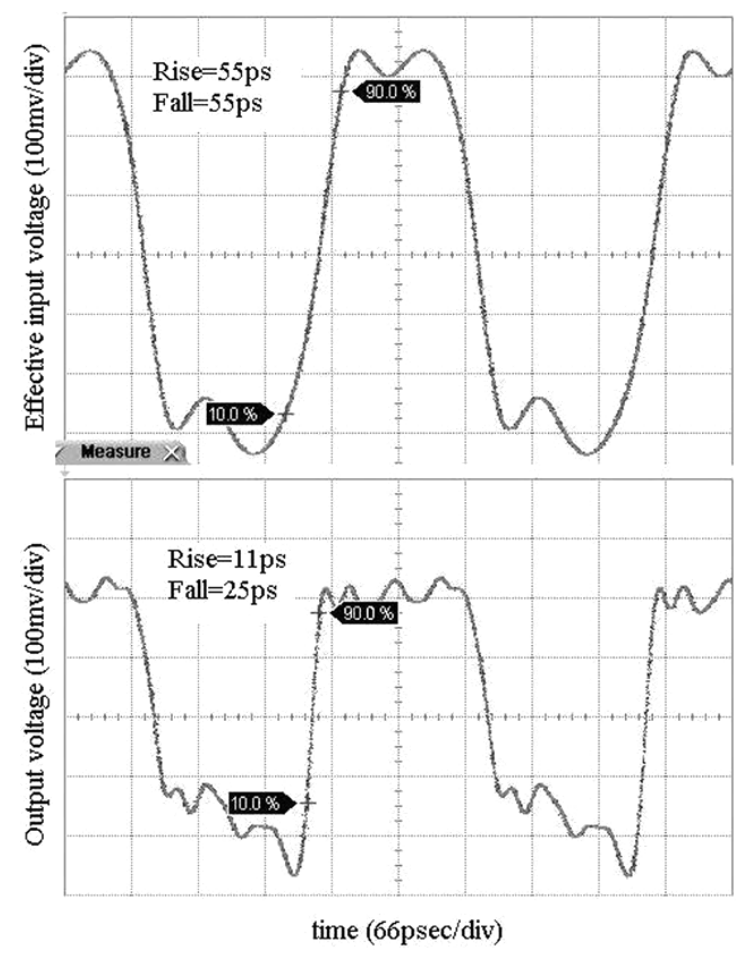

Fig. 19. Input and output waveforms of the edge sharpening line.

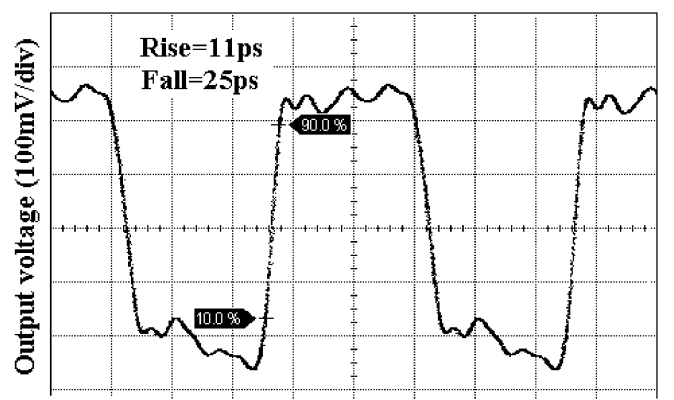

Fig. 20. Output waveforms of the edge sharpening line with different amplitude.

rowing line and rise and fall times of our edge sharpening line instead of measuring the exact values. To be accurate, we should import the frequency response of measurement system and our measured pulse width and rise/fall times to a computer simulator (like Matlab) and find out the upper limit of these parameters. In this case, computer simulations shows that the pulse width of output of pulse narrowing line and rise time of output pulse of edge sharpening line are less than $8 \mathrm{ps}$ and fall time of output pulse of edge sharpening line is around $23 \mathrm{ps}$.

\section{CONCLUSION}

We have introduced and analyzed pulse narrowing and edge sharpening passive nonlinear transmission lines, using accumulation-mode MOS varactors and the gradual scaling lines, showing simultaneous edge sharpening for both rising and falling edges in silicon. The experimental results show considerable improvement in the rise and fall times of the pulses These lines can have applications in ultra-wideband systems, broadband signal generations, and high-speed serial communications.

\section{APPENDIX}

Assuming a small $\delta$, and ignoring the high-order terms, we obtain

$$
\begin{aligned}
V_{n} \Leftrightarrow V(x)= & V \\
V_{n+1} \Leftrightarrow V(x+\delta)= & V+\delta \frac{\partial V}{\partial x}+\frac{1}{2} \delta^{2} \frac{\partial^{2} V}{\partial x^{2}}+\frac{1}{6} \delta^{3} \frac{\partial^{3} V}{\partial x^{3}} \\
& +\frac{1}{24} \delta^{4} \frac{\partial^{4} V}{\partial x^{4}}+\sum_{n=5}^{\infty} \frac{1}{n !} \delta^{n} \frac{\partial^{n} V}{\partial x^{n}} \\
V_{n-1} \Leftrightarrow V(x-\delta)= & V-\delta \frac{\partial V}{\partial x}+\frac{1}{2} \delta^{2} \frac{\partial^{2} V}{\partial x^{2}}-\frac{1}{6} \delta^{3} \frac{\partial^{3} V}{\partial x^{3}} \\
& +\frac{1}{24} \delta^{4} \frac{\partial^{4} V}{\partial x^{4}}+\sum_{n=5}^{\infty} \frac{(-1)^{n}}{n !} \delta^{n} \frac{\partial^{n} V}{\partial x^{n}} .
\end{aligned}
$$

Substituting (A1)-(A3) into (1) we obtain

$$
\ell \frac{\partial}{\partial t}\left[c(V) \frac{\partial V}{\partial t}\right]=\delta^{2} \frac{\partial^{2} V}{\partial x^{2}}+\frac{1}{12} \delta^{4} \frac{\partial^{4} V}{\partial x^{4}}
$$

\section{ACKNOWLEDGMENT}

The authors would like to thank D. Ham, H. Wu, A. Komijani, C. White, M. Sharif, M. Taghivand, H. Hashemi, S. Kee, and B. Analui of Caltech and Prof. M. Horowitz of Stanford University for helpful discussions, and M. Azarmnia for support. They also acknowledge IBM Corporation for prototype fabrication, and Agilent Technologies for test equipment support.

\section{REFERENCES}

[1] J. S. Russell, "Report on waves," in Rep. 14th Meeting of the British Association for the Advancement of Science, Sep. 1844, pp. 311-90.

[2] P. G. Drazin and R. S. Johnson, Solitons. Cambridge, U.K.: Cambridge Univ. Press, 1989.

[3] M. J. Ablowitz and H. Segur, Solitons and the Inverse Scattering Transform. Philadelphia, PA: Society for Industrial and Applied Mathematics, 1981.

[4] J. R. Tailor, Optical Solitons-Theory and Experiment. Cambridge, U.K.: Cambridge Univ. Press, 1992.

[5] R. K. Bullough and P. J. Caudrey, Solitons. Berlin, 1980: SpringerVerlag.

[6] E. Infeld and G. Rowlands, Nonlinear Waves, Solitons and Chaos. Cambridge, U.K.: Cambridge Univ. Press, 1990.

[7] P. J. Olver and D. H. Sattinger, Solitons in Physics, Mathematics, and Nonlinear Optics. New York: Springer-Verlag, 1990.

[8] M. Remoissenet, Waves Called Solitons: Concepts and Experiments. Berlin, Germany: Springer-Verlag, 1994.

[9] M. G. Case, "Nonlinear Transmission Lines for Picosecond Pulse, Impulse and Millimeter-Wave Harmonic Generation," Ph.D. dissertation, Univ. of California, Santa Barbara, 1993.

[10] M. J. W. Rodwell, M. Kamegawa, R. Yu, M. Case, E. Carman, and K Giboney, "GaAs nonlinear transmission lines for picosecond pulse generation and millimeter-wave sampling," IEEE Trans. Microwave Theory Tech., vol. 39, no. 7, pp. 1194-1204, Jul. 1991.

[11] E. Kameda, T. Matsuda, Y. Emura, and T. Ohzone, "Study of the currentvoltage characteristics in MOS capacitors with Si-implanted gate oxide," Solid-State Electron., vol. 43, no. 3, pp. 555-63, Mar. 1999.

[12] S. Matsumoto et al., "Validity of mobility universality for scaled metal-oxide-semiconductor field-effect transistors down to $100 \mathrm{~nm}$ gate length," J. Appl. Phys., vol. 92, no. 9, pp. 5228-32, Nov. 2002.

[13] L. Larcher, P. Pavan, F. Pellizzer, and G. Ghidini, "A new model of gate capacitance as a simple tool to extract MOS parameters," IEEE Trans. Electron Devices, vol. 48, no. 5, pp. 935-45, May 2001. 
[14] E. R. Benton and G. W. Platzman, "A table of solutions of the of the one-dimensional Burgers equation," Quart. Appl. Math., pp. 195-212, Jul. 1972.

[15] E. Afshari et al., "Inhomogenous transmission lines: One- and two-dimensional models," SIAM J. Appl. Math., submitted for publication.

[16] R. A. Scholtz, "Signal selection for the indoor wireless impulse radio channel," in Proc. IEEE Vehicular Technology Conf., May 1997.

[17] High Frequency Electromagnetic Software. SONNET Software. [Online]. Available: http://www.sonnetusa.com/

[18] Advanced Design System User Guide, Agilent, Palo Alto, CA.

[19] Matlab user Guide. MathWorks, Natick, MA. [Online]. Available: http://www.mathworks.com/

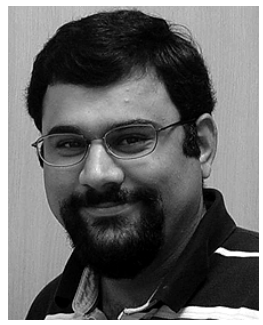

Ehsan Afshari (S'97) was born in Tehran, Iran in 1979. He received the B.S. degree in Electronics Engineering from the Sharif University of Technology, Tehran, Iran and the M.S. degree in electrical engineering from the California Institute of Technology, Pasadena, in 2003, where he is currently working toward the Ph.D. degree.

His research interest is analog integrated circuits and systems design using new technologies, ultra high speed circuit design for wireless communications.

Mr. Afshari was honored as the nation's Best Engineering Student by the President of Iran. He was also the recipient of the Best Student Paper Award at the Custom Integrated Circuits Conference (CICC), September 2003, the Best Undergraduate Paper Award at the Iranian Conference on Electrical Engineering, 1999, the recipient of the Silver Medal in the Physics Olympiad in 1997, and the recipient of the Award of Excellence in Engineering Education from the Association of Professors and Scholars of Iranian Heritage (APSIH) in May 2004.

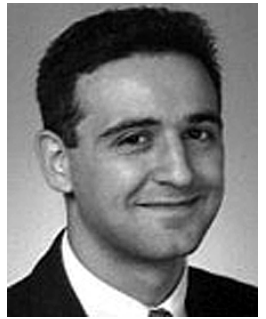

Ali Hajimiri (S'95-M'99) received the B.S. degree in electronics engineering from the Sharif University of Technology, Tehran, Iran, and the M.S. and Ph.D. degrees in electrical engineering from Stanford University, Stanford, CA, in 1996 and 1998, respectively.

He was a Design Engineer with Philips Semiconductors, where he worked on a BiCMOS chipset for GSM and cellular units from 1993 to 1994. In 1995, he was with Sun Microsystems, where he worked on the UltraSPARC microprocessor's cache RAM design methodology. During the summer of 1997, he was with Lucent Technologies, Laboratories, Murray Hill, NJ, where he investigated low-phase-noise integrated oscillators. In 1998, he joined the Faculty of the California Institute of Technology, Pasadena, where he is an Associate Professor of Electrical Engineering and the director of Microelectronics and Noise Laboratories. His research interests are high-speed and RF integrated circuits. He is a coauthor of The Design of Low Noise Oscillators (Kluwer, 1999) and holds several U.S. and European patents. He is a cofounder of Axiom Microdevices Inc.

Dr. Hajimiri is on the top 100 innovators (TR100) list. He was the Gold Medal winner of the National Physics Competition and the Bronze Medal winner of the 21st International Physics Olympiad, Groningen, Netherlands. He was a corecipient of the International Solid-State Circuits Conference (ISSCC) 1998 Jack Kilby Outstanding Paper Award and a three times winner of the IBM faculty partnership award as well as National Science Foundation CAREER award. $\mathrm{He}$ is an Associate Editor of the IEEE Journal of Solid-STATE CiRCuITS (JSSC) and a member of the Technical Program Committee of the International Solid-State Circuits Conference (ISSCC). He has also served as an Associate Editor of the IEEE TRANSACTIONS ON CIRCUITS AND SYSTEMS (TCAS) PART II, a member of the Technical Program Committees of the International Conference on Computer Aided Design (ICCAD), Guest Editor of the IEEE TRANSACTIONS ON MICROWAVE THEORY AND TECHNIQUES, and a member of the Guest Editorial Board of the Transactions of Institute of Electronics, Information and Communication Engineers of Japan (IEICE). 